\title{
High-gamma activity in the human hippocampus during inter-trial rest periods of a virtual navigation task
}

\section{Running title: Human hippocampal high-gamma during awake rest}

Yi Pu ${ }^{1,2, *}$, Brian R. Cornwell ${ }^{3}$, Douglas Cheyne ${ }^{4,5,6}$, Blake W. Johnson ${ }^{1,2}$

${ }^{1}$ ARC Centre of Excellence in Cognition and its Disorders, Macquarie University, Sydney, NSW 2109, Australia

${ }^{2}$ Department of Cognitive Science, Macquarie University, Sydney, NSW 2109, Australia

${ }^{3}$ Brain and Psychological Sciences Research Centre, Swinburne University of Technology, Melbourne, VIC 3122, Australia

${ }^{4}$ Program in Neurosciences and Mental Health, Hospital for Sick Children Research Institute, Toronto, Ontario, M5G 0A4, Canada

${ }^{5}$ Institute of Medical Sciences and Institute of Biomaterials and Biomedical Engineering, University of Toronto, Toronto, Ontario, M5S 2J7, Canada;

${ }^{6}$ Department of Medical Imaging, University of Toronto, Toronto, Ontario, M5T 1W7, Canada.

Correspondence author: Yi Pu. Address: Department of Cognitive Science, Macquarie University, Sydney, NSW 2109, Australia.

Email: yi.pu@mq.edu.au. Phone: +61 298502974 


\begin{abstract}
1 Abstract
2

3 In rodents, hippocampal cell assemblies formed during learning of a navigation task

4 are observed to re-emerge during resting (offline) periods, accompanied by

5 high-frequency oscillations (HFOs). This phenomenon is believed to reflect

6 mechanisms for strengthening newly-formed memory traces. Using

7 magnetoencephalography recordings and a beamforming source location algorithm

8 (synthetic aperture magnetometry), we investigated high-gamma $(80-140 \mathrm{~Hz})$

9 oscillations in the hippocampal region in 18 human participants during inter-trial rest

10 periods in a virtual navigation task. We found right hippocampal gamma oscillations

11 mirrored the pattern of theta power in the same region during navigation, varying as a

12 function of environmental novelty. Gamma power during inter-trial rest periods was

13 positively correlated with theta power during navigation in the first training set when

14 the environment was new and predicted faster learning in the subsequent training set

15 two where the environment became familiar. These findings provide evidence for

16 human hippocampal reactivation accompanied by high-gamma activities immediately

17 after learning and establish a link between hippocampal high-gamma activities and 18 memory consolidation.
\end{abstract}

20 Key words: Hippocampus, high-gamma oscillations, replay, virtual spatial navigation,

21 magnetoencephalography (MEG) 


\section{Introduction}

2

3

4 1989, 2015). In the encoding phase, during active exploration of an environment, a

5 transient change of synaptic strengths in the hippocampus is formed accompanied by

6 theta-band neuronal oscillations. Subsequently, during 'offline' states, including

7 slow-wave sleep and quiet wakefulness, the newly formed synaptic network

8 re-emerges, accompanied by high frequency oscillations (HFOs), operating to

9 potentiate and strengthen the synaptic changes and thereby consolidate the otherwise

10 labile memory traces.

11 environmental novelty (Carr et al., 2011): After navigating in a new environment,

19 the strength of place cell replay is stronger (Diba \& Buzsaki, 2007; O'Neill et al., 2008) and the probability of the occurrence of HFOs and the firing rates of place cells

21 are significantly higher (Cheng \& Frank, 2008) than that following navigation in a

22 familiar environment. 
The two-stage model has been intensively investigated in animal models. Are comparable neurophysiological learning mechanisms used in the human hippocampus?

3 Currently, there is very limited, but highly suggestive evidence that this is the case.

4 fMRI studies (Deuker et al., 2013; Gruber et al., 2016; Staresina et al., 2013; Tambini

5 \& Davachi, 2013; Tambini et al., 2010) have reported that brain regions that are active

6 during learning, are reactivated during sleep or rest periods after learning. For

7 instance, using multivariate pattern classification analysis, Deuker et al. (2013) found

8 stimulus-specific patterns during encoding reoccurred spontaneously during

9 postlearning resting periods and sleep.

11 To date, there was only limited electrophysiological evidence pertaining to the

12 two-stage model. For instance, from intracranial recordings in human patients,

13 Axmacher et al. (2008) reported robust high-gamma rhythms $(80-140 \mathrm{~Hz})$ during

14 the post-learning sleep period in the hippocampus and rhinal cortex, and high-gamma

15 in the rhinal cortex was positively correlated with subsequent memory performance.

16 Using noninvasive MEG measurements, Cornwell et al., (2014) reported that

17 post-learning high-gamma power was positively correlated with spatial learning

18 performance before the rest period. But in the two studies, there was no control

19 condition, it is therefore uncertain whether the high-gamma activities reported are

20 learning-specific or only a general trait marker related to general cognitive processing

21 speed. Recently, using decoding methods, Kurth-Nelson et al. (2016) found during

22 object-free periods after learning, the brain spontaneously replayed the 
1 representations of four objects learned in the learning period in a reverse order.

2 This study reveals that the replay mechanism might be a fundamental neural

3 computation in human brain as well. However, these results are on the MEG sensor

4 level and which source brain regions and neuronal oscillations are related to the

5 replay phenomenon is unknown.

In the present study, we leveraged the high time resolution of MEG to investigate

8 the temporal dynamics of human hippocampal "reactivation" during ITI immediately

9 after learning trials. MEG was recorded while participants performed two training sets

10 of a virtual Morris water maze task. Each set included a hidden platform condition

11 (task: finding the hidden platform) and a random swimming condition (task: aimlessly

12 swimming in a pool without platforms). Environment layouts of each condition in the

13 two training sets were the same. In a previous report on data from the same

14 experiment (Pu et al., 2017), we studied the role of low frequency theta oscillations (4

$15-8 \mathrm{~Hz}$ ) in spatial encoding during navigation. We found that there was significantly

16 greater theta power in right hippocampus in the first compared to the second training

17 set, which was associated with environment encoding; and there was significantly

18 more left hippocampal theta in the hidden platform condition than in the random

19 swimming condition, which was associated with encoding of the hidden platform 20 location. 
1 reflect the patterning of hippocampal theta power change during navigation, and

2 whether the power of high-gamma was correlated with the theta power, since replay is

3 proportional to previous learning in rodents (Sutherland \& McNaughton, 2000, see

4 Buzsaki, 2015 for a review). We also investigated whether high-gamma power after

5 navigating in the new environment (first training set) was associated with speed of

6 spatial learning in the familiar environment (second training set), since consolidation

7 of newly-learned environment to form a cognitive map of the space should facilitate

8 flexible navigation to new locations in the same environment (Wolbers \& Hegarty,

$92010)$.

11 Materials and Methods

13 Participants. Eighteen male participants (mean age $=29$ years; range $=18-39$ years)

14 participated in the study. Two additional participants were excluded from the final

15 data analyses because of the excessive head movement. The study was approved by

16 Macquarie University's human subjects ethics committee. All participants gave

17 written informed consent. Analysis of data during active navigation was previously

18 reported in $\mathrm{Pu}$ et al. (2017). The current analysis investigated high-gamma during the

19 ITI of the experiment when participants rested quietly following each trial of spatial 20 navigation.

22 Experiment design. A detailed description of the experimental paradigm is in Pu et al. 
1 (2017). In brief, naive participants performed two training sets of a virtual Morris

2 water maze task. In each training set of the task, there were two conditions. In the

3 hidden platform condition, participants needed to find a hidden platform submerged

4 in opaque water by using the visual cues on the walls surrounding the virtual pool. In

5 the random swimming condition, participants moved aimlessly in the same virtual

6 pool (but with no visual cues on the walls). The environment of each condition in the

7 two training sets was the same, thus the environment in the first training set was

8 defined as new environment and that in the second one as familiar environment.

9 Therefore, the difference between the two training sets allowed us to measure

10 learning of the environment ( $\mathrm{Pu}$ et al., 2017), and the difference between hidden

11 platform condition and random swimming condition provided an index of

12 goal-directed spatial navigation (Cornwell et al., 2008). To avoid the possibility that

13 environment learning was confounded with learning a specific location, the location

14 of the hidden platform was changed and counterbalanced between the training sets.

17 random swimming trials respectively, presented in alternating blocks of four trials.

18 Between each trial, there was a 4.5 - $5.5 \mathrm{~s}$ ITI (Figure 1), during which a gray screen

19 was presented and participants rested quietly without movement.

21 Behavioral measures. The length of the path taken from the starting position to the 22 hidden platform in each trial was recorded. Learning rate was computed as the 
1 average path length of the first block minus that of the last one, divided by the number

2 of blocks. To capture a more instant learning change, the slope of a linear regression

3 line fit to the average path lengths across the five blocks was computed.

5 MEG recordings. Recordings were made in a magnetically shielded room (Fujihara

6 Co. Ltd., Tokyo, Japan) with a 160-channel KIT system (Model PQ1160R-N2,

$7 \quad$ Kanazawa, Japan) with superconducting quantum interference device (SQUID)-based

8 first-order axial gradiometers (50-mm baseline; Kado et al., 1999; Uehara et al., 2003).

9 Neuromagnetic signals were digitized continuously at a sampling rate of $1000 \mathrm{~Hz}$

10 filtered at 0.03 and $200 \mathrm{~Hz}$. Before recordings, the locations of the five marker coils

11 and three fiducial markers, and the participant's head shape were digitised with a pen

12 digitizer (Polhemus Fastrack, Colchester, VT, USA). The five marker coils were 13 energized before and after each training set to determine head movement and position 14 within the MEG dewar.

16 MRI scans. High-resolution T1-weighted anatomical magnetic resonance images 17 (MRIs) were acquired in a separate session at Macquarie University Hospital, using a 18 3T Siemens Magnetom Verio scanner with a 12-channel head coil. Images were 19 obtained using 3D GR\IR scanning sequence with the following parameters: 20 repetition time, $2000 \mathrm{~ms}$; echo time, $3.94 \mathrm{~ms}$; flip angle, 9 degrees; slice thickness, $210.93 \mathrm{~mm}$; field of view, $240 \mathrm{~mm}$; image dimensions, $512 \times 512 \times 208$. 


\section{MEG analyses}

2 High-gamma during ITI. The MEG data during the ITI were epoched $(-4.5-0 \mathrm{~s} ; 0 \mathrm{~s}$

3 was the onset of the next trial; $4.5 \mathrm{~s}$ was the shortest ITI across trials) and were

4 labeled as post hidden platform condition and post random swimming condition

5 respectively. Sources were reconstructed using synthetic aperture magnetometry

6 (SAM) beamformer analysis (Hillebrand et al., 2005; Robinson \& Vrba, 1999)

7 implemented in the BrainWave toolbox (version 3.0, http://cheynelab.utoronto.ca/).

8 SAM was performed on unaveraged data so that it can identify sources that are not

9 phase-locked or time-locked activities. It estimates power changes within specific

10 frequency ranges and time windows across the whole brain without a prior

11 assumption of the number and the locations of the active source (Robinson \& Vrba, 12 1999).

MEG has been shown to reliably localize activity from the hippocampus in both simulation studies (e.g., Attal et al., 2007; Chupin et al., 2002; Meyer et al., 2017; Quraan et al., 2011; Stephen et al., 2005) and empirical experiments (e.g., Backus et

17 al., 2016; Cornwell et al., 2008; Riggs et al., 2009; Tesche \& Karhu, 2000). Recently,

18 Crespo-Garcia et al. (2016) have shown an agreement between simultaneous 19 intracranial depth recordings and MEG virtual sensor recordings of hippocampal 20 activity. 
1 general much smaller than power at lower frequencies. Nevertheless, many previous

2 studies have shown that high-gamma activity in many brain regions is successfully

3 detected by MEG (e.g., Cheyne et al., 2008; Cheyne \& Ferrari, 2013; Cornwell et al.,

4 2014; Muthukumaraswamy, 2013 for a review). The hippocampus in particular is

5 known to be a robust generator of gamma oscillations: Invasive recordings in animal

6 models have shown substantially greater power of high frequency gamma during

7 rest/sleep compared to low frequency theta during navigation (Buzsaki, 2015; Buzsaki

8 \& Silva, 2012).

9

11 volumetric grids encompassing the whole head. SAM operates by constructing an

12 adaptive spatial filter (beamformer weights) for each grid location, based on a

13 combination of lead fields calculated from the forward solution and the data

One commonly used method of normalization (e.g. Cornwell et al., 2014; Perry, 2015).

19 noise estimate (Robinson \& Vrba, 1999; Vrba \& Robinson, 2001). Another

21 normalization approach (e.g. Cornwell et al., 2012; Isabella et al., 2015) uses a 22 pseudo-F or pseudo-T metric, which computes the percentage change (pseudo-F) or 
1 absolute change (pseudo-T) of the signal power in an active state relative to a control

2 state so as to implicitly control the noise level (under the assumption that the two

3 states have similar noise levels).

In the present analyses, the source power of high-gamma activity during the ITI

6 rest period was computed using a pseudo- $Z$ metric because we were interested in

7 estimating spontaneous high-gamma power (as opposed to event-related power

8 changes). The forward model was a single sphere volume conduction model

9 (Lalancette et al., 2011; Sarvas, 1987) derived from individual MRIs. Data covariance

10 matrices were calculated for the whole epoch for the frequency band of $80-140 \mathrm{~Hz}$,

11 the same frequency range as used in Axmacher et al. (2008) and Cornwell et al.

12 (2014). Thus the length of the covariance matrix in the post hidden platform condition

13 was 20 trials $\times 4.5 \mathrm{~s} /$ trial $=90 \mathrm{~s}$ and that in the post random swimming condition was

1419 trials $\times 4.5 \mathrm{~s} /$ trial $=85.5 \mathrm{~s}$. In the latter, there were 19 trials instead of 20 , because

15 the last trial of the experiment was always a random swimming trial and the

16 experimental program aborted after the completion of the last trial. The slight

17 difference in covariance window length for post hidden platform condition and post

18 random swimming condition was not expected to significantly influence source

19 estimation: Brookes et al. (2008) demonstrated that if the bandwidths of the estimated

20 frequency band was $>50 \mathrm{~Hz}$, and when covariance window length amounted to $40 \mathrm{~s}$,

21 the accuracy of source estimation would be very high and increasing the covariance

22 window length would not greatly improve the accuracy of source estimation. Source 
1 power was estimated across the entire 3D source space at a resolution of $4 \times 4 \times 4 \mathrm{~mm}$.

3 Group statistics. The resulting volumetric SAM images were warped to a standard

4 Talairach template space and analyzed with Analysis of Functional Neuroimaging

5 (AFNI) software (Cox, 1996; http://afni.nimh.nih.gov/afni). To address the first

6 research question, i.e., whether hippocampal high-gamma during ITI mirrored the

7 pattern of hippocampal theta activity during navigation, first, we defined a region of

8 interest (ROI) in the right hippocampus/parahippocampus, in which a significant main

9 effect of training set was shown for theta power during navigation; and two ROIs

10 (because this effect occurred in two time windows: $1-2 \mathrm{~s}$ and $1.5-2.5 \mathrm{~s}$ ) in the left

11 hippocampus and parahippocampus, which showed a significant main effect of

12 condition for theta power during navigation in theta frequency band reported in $\mathrm{Pu}$ et

13 al. (2017). The mean of high-gamma power (pseudo-Z values) from the above ROIs

14 was extracted from each condition and training set. 2 (condition: post hidden platform

15 vs. post random swimming) $\times 2$ (training set: $1^{\text {st }}$ vs. $\left.2^{\text {nd }}\right)$ within-subject ANOVAs

16 were computed for the high-gamma power in the right and left hippocampi, with the

17 significance level corrected to $\mathrm{p}=0.05 / 3=0.017$ (because we did ANOVA analyses

18 three times).

20 To examine the focality of hippocampal activations and to address the possibility

21 that the effects seen in the ROIs were due to signal leakage from cortical regions, we

22 performed a 2 (condition: post hidden platform vs. post random swimming) $\times 2$ 
1 (training set: $1^{\text {st }}$ vs. $2^{\text {nd }}$ ) within-subject ANOVA for each voxel across the whole brain.

2 False positives were controlled by using a small volume FDR correction method in a

3 mask containing bilateral hippocampi and parahippocampi with the threshold of $\mathrm{p}<$

$4 \quad 0.005, \mathrm{q}<0.05$.

6 High-gamma during navigation. We also investigated whether comparable

7 high-gamma power was elicited during the navigation period. MEG data were

8 epoched into $0-4 \mathrm{~s}(0 \mathrm{~s}$ was the trial onset, $4 \mathrm{~s}$ was the fastest time from the starting

9 point to the hidden platform among all trials and participants) for each condition

10 (hidden platform and random swimming condition). Beamformer images were

11 computed for the frequency range of $80-140 \mathrm{~Hz}$ for this period. Then, the

12 standardized beamformer images were analysed with a 2 (condition: hidden platform

13 vs. random swimming) $\times 2$ (training set: 1 st vs. 2 nd) within-subject ANOVA, with the

14 significance threshold being $\mathrm{p}<0.005$, FDR corrected $\mathrm{q}<0.05$.

16 Time frequency plots. To exhibit the evolution of high-gamma power change during

17 ITI, time frequency representations (TFRs) were constructed for the peak voxel of the

18 hippocampal region from the whole brain analyses. A five-cycle wavelet was

19 convolved with the beamformed source activity over a frequency range of $1-200 \mathrm{~Hz}$

20 in $1 \mathrm{~Hz}$ steps from $-4.5-0 \mathrm{~s}$ using the formula of

$$
w\left(t, f_{0}\right)=A \exp \left(-t^{2} / 2 \sigma_{t}^{2}\right) \exp \left(2 i \pi f_{0} t\right)
$$

22 The final TFRs were presented as the power change in one condition/training set 
1 relative to the other condition/training set.

3 Correlational analyses

4 High-gamma during ITI versus subsequent navigation performance. To test the

5 hypothesis that stronger replay after spatial learning in a new environment for

6 memory consolidation of the environment is associated with quicker subsequent

7 learning in the same environment when it became more familiar, the hippocampal

8 high-gamma power which showed a significant effect in the ANOVA analyses in the

9 post hidden platform condition relative to navigation period in hidden platform

10 condition in the first training set (new environment), was correlated with the learning

11 rate in the second training set using Pearson correlation implemented in IBM SPSS

12 (version 23).

14 High-gamma during ITI versus theta during navigation. To test the hypothesis

15 that replay was proportional to encoding, the mean of the high-gamma power

16 (pseudo-Z values) in the ITI after hidden platform condition and after random

17 swimming condition in the hippocampal ROI, which showed a significant effect in the

18 ANOVA analyses, were extracted and correlated with the mean of theta power

19 (pseudo- $Z$ values) in the same ROI in the time window of $1.25-2.25$ s showing a

20 significant encoding effect in $\mathrm{Pu}$ et al. (2017) during navigation in the hidden

21 platform condition and random swimming condition respectively. The method of

22 computing pseudo- $Z$ images for theta power during navigation was the same as used 
1 for computing pseudo-Z images for high-gamma power during ITI. To determine if

2 the correlation was constrained within the hippocampus and parahippocampus, the

3 mean of high-gamma power (pseudo- $Z$ values) in the ROI was also correlated

4 voxel-wisely with pseudo-Z image of theta power during navigation across the whole

5 brain. The significance threshold was set as $\mathrm{p}<0.005$ (uncorrected).

7 Post-hoc analyses. Since the ITI was random jittered, and in the primary analyses we

8 epoched the data from $-4.5-0 \mathrm{~s}$, to confirm that the main effect of hippocampal

9 high-gamma was not influenced by the method of epoching, in the post-hoc analyses,

10 we epoched the data from the end of the trial to $4.5 \mathrm{~s}$ onward. Beamformer analyses

11 were carried out for the high-gamma power during this time period. Then a 2

12 (condition: post hidden platform vs. post random swimming) $\times 2$ (training set: 1st vs.

13 2nd) within-subject ANOVA analysis was performed for high-gamma power within

14 the hippocampal and parahippocampal ROI. We reasoned that we should get similar

15 results as those from the primary analyses, because the main time periods largely

16 overlapped for the two epochs and the brain activities we were interested were

17 spontaneous activities, which were not phase- or time-locked to any stimulus. Since

18 the SAM beamformer can capture non-phase/time locked activities, how the data was

19 epoched and time-aligned should not in principle influence the main results.

\section{Results}


1 High-gamma during ITI. There was a main effect of condition $(\mathrm{F}=6.948, \mathrm{p}<0.05$,

2 corrected, $\eta 2=0.29)$ and a main effect of training set $(F=7.85, p<0.05$, corrected,

$3 \eta 2=0.316$ ) for high-gamma power in the right hippocampal ROI during ITI (Figure

4 2A). No significant effects were found for high-gamma power in the left hippocampal

5 ROI. These results were confirmed by a whole brain 2 (conditions: post hidden

6 platform vs. post random swimming) $\times 2$ (training sets: first vs. second) repeated

7 measures ANOVA analysis, which revealed a significant main effect of training set ( $p$

$8<0.005, \mathrm{q}<0.05$, FDR corrected, peak voxel in right hippocampus, Talairach

9 coordinates: $\mathrm{x}=18 \mathrm{y}=-5 \mathrm{z}=-8)$ and a main effect of condition $(\mathrm{p}<0.005, \mathrm{q}<0.05$,

10 FDR corrected, peak voxel in right parahippocampus, Talairach coordinates: $\mathrm{x}=27 \mathrm{y}$

$11=-4 \mathrm{z}=-24$ ) (Figure 2C). No other significant effects were found in other parts of

12 hippocampi.

Time-frequency plots (Figure 3) show high-gamma power evolution during the

15 ITI in the right hippocampus. The contrasts show greater high-gamma power in the

16 right hippocampus during the ITI in the first training set (new environment) in the

17 second training set (familiar environment); and greater high-gamma power following

18 the hidden platform condition than following the random swimming condition. Visual

19 inspection revealed that the greatest high-gamma increase occurred in the time

20 window of $-2.5-0 \mathrm{~s}$ in both group-averaged TFRs (Figure 3). All these results

21 indicate that hippocampal high-gamma power exhibited the same power change

22 pattern as hippocampal theta power during navigation in the new vs. familiar 
1 environment and high-gamma power during the ITI after navigating in the hidden

2 platform condition which required more learning was stronger than that after

3 navigating in the random swimming condition where learning requirement was much

4 lower.

6 High-gamma during navigation and its comparison with high-gamma during ITI.

7 No significant results were found for high-gamma power during navigation (brain

8 images not shown. no single voxel was found in the hippocampi and parahippocampi

9 even when $\mathrm{p}=0.05$, uncorrected). Direct comparison of high-gamma power during

10 ITI and that during navigation in the right hippocampal ROI showed that high-gamma

11 power in the post hidden platform condition during ITI in the first training set was

12 significant higher $(\mathrm{t}(17)=3.072, \mathrm{p}=0.007$, cohen's $\mathrm{d}=0.74$, Figure 2 A \& Figure $2 \mathrm{~B}$ )

13 than that during navigation in the hidden platform condition in the first training set.

14 No significant difference was found for the second training set $(\mathrm{t}(17)=1.000, \mathrm{p}=$

15 0.329), indicating that hippocampal high-gamma increase during ITI was most

16 apparent following navigation in the new environment.

20 condition in both training sets $(\mathrm{t}(17)=1.66, \mathrm{p}=0.114$ for the first training set; $\mathrm{t}(17)=$

$21-0.61, p=0.548$ for the second training set). This might suggest although after

22 random swimming condition, right hippocampal high-gamma power showed the same 
1 pattern of right hippocampal theta power during navigation, the 'replay' effect during

2 the ITI following navigation in a condition with low learning demands was not as

3 salient as that following navigation in a condition with high learning demands

4 (Eschenko \& Sara, 2008; Girardeau et al., 2014). This conclusion was supported by

5 the result of direct comparison between the high-gamma power difference between

6 post hidden platform and hidden platform condition (Diff_H) in the first training set

7 and the high-gamma power difference between post random swimming and random

8 swimming condition (Diff_R) in the first training training set, which showed that the

9 Diff_H in the first training set was significantly larger than the Diff_R in the first

10 training $\operatorname{set}(\mathrm{t}(17)=2.264, \mathrm{p}=0.037$, cohen's $\mathrm{d}=0.589)$.

\section{Correlation Results}

13 Right hippocampal high-gamma during ITI vs. navigation performance.

14 Consistent with our hypothesis that the more the hippocampal high-gamma power

15 increase was during the ITI after encoding the new environment, the faster the

16 participant would learn a new location in the subsequent training set when the

17 environment had become familiar, we found a significant correlation between the

18 power increase of high-gamma during the ITI after hidden platform condition relative

19 to that during navigation period in the hidden platform condition in the right

20 hippocampal ROI in the first training set with learning rate in the second training set

$21(\mathrm{r}=0.601, \mathrm{p}=0.004$, one-tailed Figure $4 \mathrm{~A})$. We also found a significant correlation

22 between the hippocampal high-gamma increase in the right hippocampal ROI with the 
1 slope of the regression line which fit the path lengths across blocks $(r=-0.418, p=$

20.042 , one-tailed. Figure not shown). The significant correlations indicate navigators

3 who showed higher high-gamma power during rest period after spatial learning in the

4 new environment learned more quickly a new hidden platform location in the same

5 environment. This is in line with results from previous literature that high-gamma

6 during rest period after learning is associated with better subsequent memory

7 performance (Axmacher et al., 2008).

9 To exclude the possibility that the correlation was not learning specific and only

10 reflected a general relationship between high-gamma power and the general cognitive

11 processing speed across subjects, we also correlated the learning rate in the second

12 training set in the hidden platform condition with the right hippocampal high-gamma

13 power change during the ITI after the hidden platform condition relative to that in the

14 hidden platform condition in the ROI in the second training set, when learning

15 requirement decreased indexed by improved navigation performance in the second

16 training set as shown in $\mathrm{Pu}$ et al. (2017). No significant correlation was found $(\mathrm{r}=$

$170.057, \mathrm{p}=0.411$, one-tailed, for the correlation between hippocampal power and

18 learning rate, $\mathrm{r}=0.029, \mathrm{p}=0.455$, one-tailed, for the correlation between

19 hippocampal power and the slope of the regression line. Figure 4B). This result

20 indicates that the correlation between high-gamma power increase after learning a

21 new environment and subsequent learning performance in the familiar environment is

22 functionally relevant. 
2 Right hippocampal high-gamma during ITI vs. right hippocampal theta during

3 navigation. Consistent with the hypothesis that replay is proportional to encoding, we

4 found a significant correlation $(\mathrm{r}=0.406, \mathrm{p}=0.046$, one tailed) between right

5 hippocampal high-gamma power during the ITI after navigating in the hidden

6 platform condition in the first training set and right hippocampal theta power during

7 navigation. Since high-gamma power increase was most salient in the time window of

$8-2.5-0 \mathrm{~s}$ in the group-averaged TFRs shown in Figure 3, we reasoned the correlation

9 between the right hippocampal high-gamma power in this time window with theta

10 power during navigation would be even stronger. As expected, the correlation was

11 significant with higher correlation coefficient $(r=0.53, p=0.017$, one-tailed, Figure

12 5A). Voxel wise correlation analysis confirmed that the significant effect was focal

13 with the local maximum in the right hippocampus (Talairach coordinate of the peak

14 voxel: $x=26, y=-17, z=-8$, Figure $5 C$ ). These significant correlations support the

15 hypothesis that 'replay' is proportional to encoding. after random swimming condition in the first training set and theta power in random

19 swimming condition in the first training set $(\mathrm{r}=0.065, \mathrm{p}=0.399$, one-tailed, Figure

20 5B). Voxel wise correlation did not yield significant correlation in any voxels in the

21 bilateral hippocampi as well. These results may indicate that the degree of faithfulness

22 of 'replay' following spatial navigation in a simple environment with low encoding 
1 requirements is low, which is in agreement with findings from animal literature

2 (Kentros et al., 2004) that faithful retrieval of a mouse's hippocampal representation

3 of an environment increased as task demands increased.

4

6 significant correlation was found between high-gamma power during ITI in the

7 second training set and theta power during navigation in the second training set for

8 both conditions $(\mathrm{r}=0.078, \mathrm{p}=0.379$, one-tailed, for correlation between high-gamma

9 power in the post hidden platform condition and theta power during navigation in the

10 second training set and theta power during navigation in the hidden platform

11 condition in the second training set; $r=0.21 \mathrm{p}=0.201$, one-tailed, for correlation

12 between high-gamma power in the post random swimming condition and theta power

13 during navigation in the random swimming condition in the second training set;),

14 suggesting that the significant correlation seen above is contingent on learning.

15

16 Post-hoc analyses. Similar effects were found for the hippocampal high-gamma

17 power during the epoched time from the end of the navigation trial to the $4.5 \mathrm{~s}$

18 forwards as for the high-gamma power epoched from $-4.5-0 \mathrm{~s}$ in the primary

19 analyses above, confirming that the significant effects of high-gamma power during

20 ITI were not influenced by the epoching method.

\section{Discussion}


Using MEG and a virtual Morris water maze task, we investigated whether

3 human hippocampal high-gamma power exhibited the same power change pattern as

4 theta rhythms during navigation. We found first, that hippocampal high-gamma power

5 after navigation mirrored the power change pattern of hippocampal theta oscillations

6 during navigation: After navigation in the new environment, hippocampal

7 high-gamma power was significantly stronger than that after navigation in the familiar

8 one, and the hippocampal high-gamma power after navigating in the hidden platform

9 condition where learning requirement was higher than that after navigation in the

10 random swimming condition with much lower learning requirement; Second, that

11 right hippocampal high-gamma power during ITI was correlated with right

12 hippocampal theta power during navigation; Third that, higher right hippocampal

13 high-gamma power after navigation in the first training set where the environment

14 was new correlated with faster learning in the second training set where the

15 environment became familiar.

18 exhibited the same power change pattern as right hippocampal theta power during

19 navigation is in line with the prediction of two-stage model for memory formation

20 (Buzsaki, 2015) and animal studies (Ambrose et al., 2016; Davidson et al., 2009;

21 Dupret et al., 2010; Jackson et al., 2006; Jadhav et al., 2012; Karlsson \& Frank, 2009;

22 Singer \& Frank, 2009, refer to Roumis \& Frank, 2015 for a review) that the 
1 hippocampus is spontaneously reactivated during rest/sleep period accompanied by

2 HFOs to promote neuronal plasticity and stabilize the newly-formed memory traces.

3 Our finding of greater high-gamma power in the ITI after exposure to the new

4 environment than in the ITI following the familiar environment is consistent with

5 reports from animal studies that HFOs after navigation in a new environment are

6 stronger, occur more frequently and are more easily detected (Foster \& Wilson, 2006;

7 O'Neill et al., 2008; Cheng \& Frank, 2008; Csicsvari et al., 2007).

9 Our data further showed no significant high-gamma power during navigation

10 period, when the theta rhythm was prominent $(\mathrm{Pu}$ et al. (2017). In addition,

11 high-gamma power after navigation in hidden platform condition in the new

12 environment was significantly greater than that during navigation in hidden platform

13 condition in the new environment, which is corroborated by the idea that relative to

14 navigation period, HFOs are more salient during the rest period when animals are

15 disengaged from the external environment (Buzsaki, 2015). In the ITI after navigation

16 in the familiar environment, with decreased learning requirements (indexed by

17 improved navigation performance shown in $\mathrm{Pu}$ et al., 2017), no high-gamma power

18 increase was found. These results suggest that high-gamma power increase is

19 modulated by environmental novelty and learning requirements (Cheng \& Frank,

20 2008). Moreover, although high-gamma power during ITI after random swimming

21 trials showed a power change in the new vs. familiar environment, high-gamma

22 increase following navigating in hidden platform trials during ITI relative to that 
1 during navigation in hidden platform trials in the new environment was significantly

2 larger than high-gamma increase after navigating in random swimming trials during

3 ITI relative to that during navigation in random swimming trials. This indicates that

4 high-gamma power during rest period are stronger after navigation in a more complex

5 environment with higher learning requirements, in agreement with the finding that

6 replay strengths vary as a function of task demands (Eschenko \& Sara, 2008;

$7 \quad$ Girardeau et al., 2014).

9 The present results complement previous studies (Cornwell et al., 2014;

10 Axmacher et al., 2008) by showing that the same hippocampal region used for

11 encoding accompanied by low frequency oscillation exhibited a similar power change

12 pattern accompanied by high-gamma oscillations during ITI after encoding, thus

13 supporting the role of hippocampal high-gamma in replay of newly learned

14 experience. These results also support previous fMRI studies (Deuker et al., 2013;

15 Gruber et al., 2016; Peigneux et al., 2006; Staresina et al., 2013; Tambini \& Davachi,

16 2013; Tambini et al., 2010; Vincent et al., 2006), which showed experience-dependent

17 reactivation during wakefulness or sleep and provides a neurophysiological

18 mechanism underlying the reactivation. The current results may also help explain why

19 some fMRI studies have not found hippocampal reactivation during rest/sleep period

20 after learning (e.g., Deuker et al., 2013, they found visual area showing a "replay"

21 effect instead of the hippocampus). Because as shown in our data, hippocampal

22 reactivation occurs immediately after each learning trial and decays over learning, 
1 during which information is being transferred to neocortex (Colgin, 2016; Kudrimoti

2 et al., 1999). Therefore, hippocampal reactivation might not be as strong and salient

3 during rest/sleep after a long period of learning as that immediately after each

4 learning trials on a faster timing scale. Further study could investigate how persistent

5 high-gamma-related hippocampal replay is.

HFOs measured using MEG are easily confounded by muscle artifacts. However,

8 source localization algorithms based on spatial filters can differentiate cognitive

9 processing source from cortical or subcortical areas with artifactual sources (Dalal et

10 al., 2011). Several checks support our contention the high-gamma effects observed in

11 the current study are not artefactual. First, the analyses were performed during the

12 inter-trial period when participants were instructed to rest quietly and to minimize

13 movement. Second, there was no significant high-gamma effect during active

14 navigation epochs which are more likely to be contaminated by muscle artifacts

15 because participants pressed buttons to move in the virtual pool. Third, the spatial 16 map of the effect was focal and localized unilaterally to right hippocampus, and TFRs

17 showed relatively narrow band power changes. Muscle artifact, in contrast, tends to 18 span large spatial regions and broad frequency ranges (from 30 to $200 \mathrm{~Hz}$ or higher;

19 Muthukumaraswamy, 2013).

21 Consistent with our hypothesis that replay is proportional to encoding, our data 22 showed that high-gamma power during the ITI after navigating in the hidden platform 
1 condition in the new environment was positively associated with theta power during

2 navigation in hidden platform condition in the new environment, when learning

3 requirement was strongest. Together with the finding that the same hippocampal

4 region used for encoding was reactivated during rest period, the correlation provides

5 further evidence that high-gamma during rest is modulated by previous learning

6 experience to accurately reinforce the newly-formed labile memory traces (Davidson

7 et al., 2009; Skaggs \& McNaughton, 1996; Wilson \& McNaughton, 1994). A

8 comparable correlation was not found for familiar environments with decreased

9 learning requirements, indicating that the correlation seen in the new environment is

10 learning induced, rather than an intrinsic relationship between gamma and theta power.

11 The random swimming condition, where learning requirements were low, showed no

12 significant correlation between theta and high-gamma in both training sets, suggesting

13 although replay is automatic, the degree of replay faithfulness may vary as a function

14 of encoding requirement. This is corroborated by the findings of a rodent study

15 (Kentros et al., 2004), showing that the faithful retrieval of a mouse's hippocampal

16 representation of an environment increases as task demands increase and place cell

17 stability tightly covaries with attention to the available spatial cues.

Further, we observed that right hippocampal high-gamma power increase

20 following hidden platform condition in the first training set relative to that during

21 navigation in this condition in the same training set correlated with learning rate in the

22 hidden platform condition in the second training set. This correlation provides strong 
1 evidence for the argument that the functional role of right hippocampal high-gamma

2 reactivation is memory consolidation. Navigators with higher right hippocampal

3 high-gamma power during the ITI after navigation in a new environment learned

4 more quickly a new location of the hidden platform in the second training set where

5 the environment was the same and become familiar, indicating right hippocampal

6 high-gamma power is related to consolidation of the newly learnt environment. Good

7 consolidation of the environment to form a cognitive map of the surroundings can

8 facilitate flexible navigation to any place in the same environment (Wolbers \&

9 Hegarty, 2010). This correlation is also consistent with observations from human

10 fMRI studies showing that reactivation strength of the hippocampus predicts

11 subsequent memory performance (Bergmann et al., 2012; Peigneux et al., 2006). The

12 direction of the correlation is also consistent with the results of Axmacher et al. (2008)

13 and Cornwell et al. (2014), documenting that stronger high-gamma power

14 corresponded to better memory performance. No correlation was found between

15 high-gamma power in the second training set (where consolidation requirement

16 decreased) with learning rate in this training set, indicating the significant correlation

17 seen above is learning specific.

19 It is noteworthy that the effects described in the current study were confined to

20 right hippocampus. In our previous findings, navigation-related theta effects were

21 found in both left hippocampus (implicated in binding the external cues to the 22 platform location) and right hippocampus (associated with encoding the environment 
1 to form the cognitive map of the space). While non-significant results do not confirm

2 the null hypothesis, this is consistent with the notion that replay is selective, such that

3 not every aspect of learning would be replayed (Deuker et al., 2013). However, the

4 mechanism underlying this replay selection is still unclear. Reactivation during awake

5 rest and sleep is more complicated than expected (Buzsaki, 2015). O'Neill et al. (2006)

6 has shown the replay might not necessarily be location dependent. Recent studies

7 (Gupta et al., 2010; Wu \& Foster, 2014) have pointed out the view that the function of

8 replay is helping construct a Tolmanian cognitive map of the environment, which

9 would result in flexible routes to the goal location on subsequent trials. This might

10 help explain why only the right hippocampus region, which is associated with

11 environmental learning was reactivated in our data. Also, the present work provide

12 new insights into the reason why right hippocampus is believed to be important in

13 spatial cognition in general (Cornwell et al., 2010; Jacobs et al., 2009; Maguire et al.,

14 1998; Nedelska et al., 2012). More research is needed in the future to investigate the

15 selective nature of hippocampal replay.

19 learning, human hippocampal high-gamma activity is evident and plays an important

20 role in replay of the newly-learned information. These findings advance our

21 understanding of the neurophysiological mechanisms and timing of hippocampal

22 reactivation after learning for memory consolidation in humans. 
1 Acknowledgements: This work was supported by Australian Research Council

2 Grants CE110001021, DP1096160. The first author was financially supported by the

3 China Scholarship Council and Macquarie University. We thank Dr. Suresh

4 Muthukumaraswamy for helpful discussions about muscle artifacts and gamma

5 activity. The authors declare no competing financial interests.

6

7

8 Correspondence author: Yi Pu. Address: Department of Cognitive Science,

9 Macquarie University, Sydney, NSW 2109, Australia.

10 Email: yi.pu@mq.edu.au. Phone: +61 298502974

11

12

13 


\section{References}

Ambrose, R. E., Pfeiffer, B. E., \& Foster, D. J. (2016). Reverse Replay of Hippocampal Place Cells Is Uniquely Modulated by Changing Reward. Neuron, 91(5), 1124-1136. doi:10.1016/j.neuron.2016.07.047

Attal, Y., Bhattacharjee, M., Yelnik, J., Cottereau, B., Lefevre, J., Okada, Y., . . . Baillet, S. (2007). Modeling and Detecting Deep Brain Activity with MEG \& EEG. Conf Proc IEEE Eng Med Biol Soc, 4937-4940.

Axmacher, N., Elger, C. E., \& Fell, J. (2008). Ripples in the medial temporal lobe are relevant for human memory consolidation. Brain, 131(Pt 7), 1806-1817. doi:10.1093/brain/awn103

Backus, A. R., Schoffelen, J. M., Szebenyi, S., Hanslmayr, S., \& Doeller, C. F. (2016). Hippocampal-Prefrontal Theta Oscillations Support Memory Integration. Curr Biol, 26(4), 450-457. doi:10.1016/j.cub.2015.12.048

Bergmann, T. O., Molle, M., Diedrichs, J., Born, J., \& Siebner, H. R. (2012). Sleep spindle-related reactivation of category-specific cortical regions after learning face-scene associations. Neuroimage, 59(3), 2733-2742. doi:10.1016/j.neuroimage.2011.10.036

Brookes, M. J., Vrba, J., Robinson, S. E., Stevenson, C. M., Peters, A. M., Barnes, G. R., . . . Morris, P. G. (2008). Optimising experimental design for MEG beamformer imaging. Neuroimage, 39(4), 1788-1802. doi:10.1016/j.neuroimage.2007.09.050

Buzsaki, G. (1989). Two-stage model of memory trace formation: a role for "noisy" brain states Neuroscience, 31(3), 551-570.

Buzsaki, G. (2015). Hippocampal sharp wave-ripple: A cognitive biomarker for episodic memory and planning. Hippocampus, 25(10), 1073-1188. doi:10.1002/hipo. 22488

Buzsaki, G., \& Silva, F. L. (2012). High frequency oscillations in the intact brain. Prog Neurobiol, 98(3), 241-249. doi:10.1016/j.pneurobio.2012.02.004

Carr, M. F., Jadhav, S. P., \& Frank, L. M. (2011). Hippocampal replay in the awake state: a potential substrate for memory consolidation and retrieval. Nat Neurosci, 14(2), 147-153. doi:10.1038/nn.2732

Cheng, S., \& Frank, L. M. (2008). New experiences enhance coordinated neural 
activity in the hippocampus. Neuron, 57(2), 303-313. doi:10.1016/j.neuron.2007.11.035

Cheyne, D., Bells, S., Ferrari, P., Gaetz, W., \& Bostan, A. C. (2008). Self-paced movements induce high-frequency gamma oscillations in primary motor cortex. Neuroimage, 42(1), 332-342. doi:10.1016/j.neuroimage.2008.04.178

Cheyne, D., \& Ferrari, P. (2013). MEG studies of motor cortex gamma oscillations: evidence for a gamma "fingerprint" in the brain? Front Hum Neurosci, 7, 575. doi:10.3389/fnhum.2013.00575

Chupin, M., Baillet, S., Okada, Y. C., Hasboun, D., \& Garnero, L. (2002). On the detection of hippocampus activity with MEG. Paper presented at the 13th International Conference on Biomagnetism, Germany.

Colgin, L. L. (2016). Rhythms of the hippocampal network. Nat Rev Neurosci, 17(4), 239-249. doi:10.1038/nrn.2016.21

Cornwell, B. R., Arkin, N., Overstreet, C., Carver, F. W., \& Grillon, C. (2012). Distinct contributions of human hippocampal theta to spatial cognition and anxiety. Hippocampus, 22(9), 1848-1859. doi:10.1002/hipo.22019

Cornwell, B. R., Johnson, L. L., Holroyd, T., Carver, F. W., \& Grillon, C. (2008). Human hippocampal and parahippocampal theta during goal-directed spatial navigation predicts performance on a virtual Morris water maze. $J$ Neurosci, 28(23), 5983-5990. doi:10.1523/JNEUROSCI.5001-07.2008

Cornwell, B. R., Overstreet, C., \& Grillon, C. (2014). Spontaneous fast gamma activity in the septal hippocampal region correlates with spatial learning in humans. Behav Brain Res, 261, 258-264. doi:10.1016/j.bbr.2013.12.031

Cornwell, B. R., Salvadore, G., Colon-Rosario, V., Latov, D. R., Holroyd, T., Carver, F. W., . . Grillon, C. (2010). Abnormal hippocampal functioning and impaired spatial navigation in depressed individuals: evidence from whole-head magnetoencephalography. Am J Psychiatry, 167(7), 836-844. doi:10.1176/appi.ajp.2009.09050614

Cox, R. W. (1996). AFNI: software for analysis and visualization of functional magnetic resonance neuroimages. Comput Biomed Res, 29, 162-173. doi:10.1006/cbmr.1996.0014

Crespo-Garcia, M., Zeiller, M., Leupold, C., Kreiselmeyer, G., Rampp, S., Hamer, H. M., \& Dalal, S. S. (2016). Slow-theta power decreases during item-place 
encoding predict spatial accuracy of subsequent context recall. Neuroimage, 142, 533-543. doi:10.1016/j.neuroimage.2016.08.021

Csicsvari, J., O'Neill, J., Allen, K., \& Senior, T. (2007). Place-selective firing contributes to the reverse-order reactivation of CA1 pyramidal cells during sharp waves in open-field exploration. Eur $J$ Neurosci, 26(3), 704-716. doi:10.1111/j.1460-9568.2007.05684.x

Dalal, S., Vidal, J. R., Hamame, C. M., Ossamdpm, T., Betrand, O., Lachaux, J. P., \& Jerbi, K. (2011). Spanning the rieh spectrum of the human brain: slow waves to gamma and beyond. Brain Struct Funct, 216, 77-84. doi:10.1007/s00429-011-0307-z

Davidson, T. J., Kloosterman, F., \& Wilson, M. A. (2009). Hippocampal replay of $\begin{array}{llll}\text { extended } & \text { experience. }\end{array}$ doi:10.1016/j.neuron.2009.07.027

Deuker, L., Olligs, J., Fell, J., Kranz, T. A., Mormann, F., Montag, C., . . . Axmacher, N. (2013). Memory consolidation by replay of stimulus-specific neural activity. J Neurosci, 33(49), 19373-19383. doi:10.1523/JNEUROSCI.0414-13.2013

Diba, K., \& Buzsaki, G. (2007). Forward and reverse hippocampal place-cell sequences during ripples. Nat Neurosci, 10(10), 1241-1242. doi:10.1038/nn1961

Dupret, D., O'Neill, J., Pleydell-Bouverie, B., \& Csicsvari, J. (2010). The reorganization and reactivation of hippocampal maps predict spatial memory performance. Nat Neurosci, 13(8), 995-1002. doi:10.1038/nn.2599

Ego-Stengel, V., \& Wilson, M. A. (2010). Disruption of ripple-associated hippocampal activity during rest impairs spatial learning in the rat. Hippocampus, 20(1), 1-10. doi:10.1002/hipo.20707

Eschenko, O., \& Sara, S. J. (2008). Learning-dependent, transient increase of activity in noradrenergic neurons of locus coeruleus during slow wave sleep in the rat: brain stem-cortex interplay for memory consolidation? Cereb Cortex, 18(11), 2596-2603. doi:10.1093/cercor/bhn020

Foster, D. J., \& Wilson, M. A. (2006). Reverse replay of behavioural sequences in hippocampal place cells during the awake state. Nature, 440(7084), 680-683. doi: $10.1038 /$ nature 04587

Girardeau, G., Benchenane, K., Wiener, S. I., Buzsaki, G., \& Zugaro, M. B. (2009). 
Selective suppression of hippocampal ripples impairs spatial memory. Nat Neurosci, 12(10), 1222-1223. doi:10.1038/nn.2384

Girardeau, G., Cei, A., \& Zugaro, M. (2014). Learning-induced plasticity regulates hippocampal sharp wave-ripple drive. J Neurosci, 34(15), 5176-5183. doi:10.1523/JNEUROSCI.4288-13.2014

Gruber, M. J., Ritchey, M., Wang, S. F., Doss, M. K., \& Ranganath, C. (2016). Post-learning Hippocampal Dynamics Promote Preferential Retention of Rewarding Events. $\quad$ Neuron, $\quad$ 89(5), 1110-1120. doi:10.1016/j.neuron.2016.01.017

Gupta, A. S., van der Meer, M. A., Touretzky, D. S., \& Redish, A. D. (2010). Hippocampal replay is not a simple function of experience. Neuron, 65(5), 695-705. doi:10.1016/j.neuron.2010.01.034

Hillebrand, A., Singh, K. D., Holliday, I. E., Furlong, P. L., \& Barnes, G. R. (2005). A new approach to neuroimaging with magnetoencephalography. Hum Brain Mapp, 25(2), 199-211. doi:10.1002/hbm.20102

Isabella, S., Ferrari, P., Jobst, C., Cheyne, J. A., \& Cheyne, D. (2015). Complementary roles of cortical oscillations in automatic and controlled processing during rapid serial tasks. Neuroimage, 118, 268-281. doi:10.1016/j.neuroimage.2015.05.081

Jackson, J. C., Johnson, A., \& Redish, A. D. (2006). Hippocampal sharp waves and reactivation during awake states depend on repeated sequential experience. $J$ Neurosci, 26(48), 12415-12426. doi:10.1523/JNEUROSCI.4118-06.2006

Jacobs, J., Lorolev, I. O., Caplan, J. B., Ekstrom, A., Litt, B., Baltuch, G., . . Kahana, M. J. (2009). Right-lateralized brain oscillations in human spatial navigation. $J$ Cogn Neurosci, 22(5), 824-836. doi:10.1162/jocn.2009.21240

Jadhav, S. P., Kemere, C., German, P. W., \& Frank, L. M. (2012). Awake hippocampal sharp-wave ripples support spatial memory. Science, 336(6087), 1454-1458. doi: $10.1126 /$ science. 1217230

Kado, H., Higuchi, M., Shimogawara, M., Haruta, Y., Adachi, Y., Kawai, J., \& Uehara, G. (1999). Magnetoencephalogram systems developed at KIT. IEEE Trans. Appl. Supercond, 9, 4057-4062. doi:doi:10.1109/77.783918

Karlsson, M. P., \& Frank, L. M. (2009). Awake replay of remote experiences in the hippocampus. Nat Neurosci, 12(7), 913-918. doi:10.1038/nn.2344 
1 Kentros, C. G., Agnihotri, N. T., Streater, S., Hawkins, R. D., \& Kandel, E. R. (2004). Increased attention to spatial context increases both place field stability and spatial memory. Neuron, 42, 283-295. doi:10.1016/S0896-6273(04)00192-8

Kudrimoti, H. S., Barnes, C. A., \& McNaughton, B. L. (1999). Reactivation of hippocampal cell assemblies: effects of behavioral state, experience, and EEG dynamics. J Neurosci, 19(10), 4090-4101.

Kurth-Nelson, Z., Economides, M., Dolan, R. J., \& Dayan, P. (2016). Fast Sequences of Non-spatial State Representations in Humans. Neuron, 91(1), 194-204. doi:10.1016/j.neuron.2016.05.028

Lalancette, M., Quraan, M., \& Cheyne, D. (2011). Evaluation of multiple-sphere head models for MEG source localization. Phys Med Biol, 56(17), 5621-5635. doi:10.1088/0031-9155/56/17/010

Maguire, E. A., Burgess, N., Donnett, J. G., Frackowiak, R. S. J., Frith, C. D., \& O'Keefe, J. (1998). Knowing where and geeting there: a huma navigation network. Science, 280, 921-924.

Meyer, S. S., Rossiter, H., Brookes, M. J., Woolrich, M. W., Bestmann, S., \& Barnes, G. R. (2017). Using generative models to make probabilistic statements about hippocampal engagement in MEG. Neuroimage. doi:10.1016/j.neuroimage.2017.01.029

Muthukumaraswamy, S. D. (2013). High-frequency brain activity and muscle artifacts in MEG/EEG: a review and recommendations. Front Hum Neurosci, 7, 138. doi:10.3389/fnhum.2013.00138

Nedelska, Z., Andel, R., Laczo, J., Vlcek, K., Horinek, D., Lisy, J., . . Hort, J. (2012). Spatial navigation impairment is proportional to right hippocampal volume. Proc Natl Acad Sci U S A, 109(7), 2590-2594. doi:10.1073/pnas.1121588109

O'Neill, J., Pleydell-Bouverie, B., Dupret, D., \& Csicsvari, J. (2010). Play it again: reactivation of waking experience and memory. Trends Neurosci, 33(5), 220-229. doi:10.1016/j.tins.2010.01.006

O'Neill, J., Senior, T., \& Csicsvari, J. (2006). Place-selective firing of CA1 pyramidal cells during sharp wave/ripple network patterns in exploratory behavior. Neuron, 49(1), 143-155. doi:10.1016/j.neuron.2005.10.037

O'Neill, J., Senior, T. J., Allen, K., Huxter, J. R., \& Csicsvari, J. (2008). Reactivation of experience-dependent cell assembly patterns in the hippocampus. Nat 
Neurosci, 11(2), 209-215. doi:10.1038/nn2037

Peigneux, P., Orban, P., Balteau, E., Degueldre, C., Luxen, A., Laureys, S., \& Maquet, P. (2006). Offline persistence of memory-related cerebral activity during active wakefulness. PLoS Biol, 4(4), e100. doi:10.1371/journal.pbio.0040100

Perry, G. (2015). The effects of cross-orientation masking on the visual gamma response in humans. Eur $J$ Neurosci, 41(11), 1484-1495. doi:10.1111/ejn.12900

Pu, Y., Cornwell, B. R., Cheyne, D., \& Johnson, B. W. (2017). The functional role of human right hippocampal/parahippocampal theta rhythm in environmental encoding during virtual spatial navigation. Hum Brain Mapp, 38(3), 1347-1361. doi:10.1002/hbm.23458

Quraan, M. A., Moses, S. N., Hung, Y., Mills, T., \& Taylor, M. J. (2011). Detection and localization of hippocampal activity using beamformers with MEG: a detailed investigation using simulations and empirical data. Hum Brain Mapp, 32(5), 812-827. doi:10.1002/hbm.21068

Riggs, L., Moses, S. N., Bardouille, T., Herdman, A. T., Ross, B., \& Ryan, J. D. (2009). A complementary analytic approach to examining medial temporal lobe sources using magnetoencephalography. Neuroimage, 45(2), 627-642. doi:10.1016/j.neuroimage.2008.11.018

Robinson, S. E., \& Vrba, J. (1999). Functional neuroimaging by synthetic aperture magnetometry (SAM) (T. Yoshimoto, M. Kotani, S. Kuriki, H. Karibe, \& N. Nakasato Eds.). Sendai, Japan: Tohoku UP.

Roumis, D. K., \& Frank, L. M. (2015). Hippocampal sharp-wave ripples in waking and sleeping states. Curr Opin Neurobiol, 35, 6-12. doi:10.1016/j.conb.2015.05.001

Sarvas, J. (1987). Basic mathmatical and electromagnetic concepts of the biomagnetic inverse problem. Phys Med Biol, 32, 11-22.

Singer, A. C., \& Frank, L. M. (2009). Rewarded outcomes enhance reactivation of experience in the hippocampus. Neuron, 64(6), 910-921. doi:10.1016/j.neuron.2009.11.016

Skaggs, W. E., \& McNaughton, B. L. (1996). Replay of neuronal firing sequences in rat hippocampus during sleep following spatial experience. Science, 271, 1870-1873. 
1 Staresina, B. P., Alink, A., Kriegeskorte, N., \& Henson, R. N. (2013). Awake reactivation predicts memory in humans. Proc Natl Acad Sci U S , 110(52), 21159-21164. doi:10.1073/pnas.1311989110

Stephen, J. M., Ranken, D. M., Aine, C. J., Weisend, M. P., \& Shih, J. J. (2005). Differentiability of simulated MEG hippocampal, medial temporal and neocortical temporal epileptic spike activity. J Clin Neurophysiol, 22, 388-401. doi:10.1097/01.WNP.0000172141.26081.78

Sutherland, G. R., \& McNaughton, B. L. (2000). Memory trace reactivation in hippocampal and neocortical neuronal ensembles. Curr Opin Neurobiol, 10, 180-186.

Tambini, A., \& Davachi, L. (2013). Persistence of hippocampal multivoxel patterns into postencoding rest is related to memory. Proc Natl Acad Sci U S A, 110(48), 19591-19596. doi:10.1073/pnas.1308499110

Tambini, A., Ketz, N., \& Davachi, L. (2010). Enhanced brain correlations during rest are related to memory for recent experiences. Neuron, 65(2), 280-290. doi:10.1016/j.neuron.2010.01.001

Tesche, C. D., \& Karhu, J. (2000). Theta oscillations index human hippocampal activation during a working memory task. Proc Natl Acad Sci U S A, 97(2), 919-924.

Uehara, G., Adachi, Y., Kawai, J., Shimogawara, M., Higuchi, M., Haruta, Y., \& Kado, H. (2003). Multi-channel SQUID systems for biomagnetic measurement. IEICE Trans Electron, E86c(43-54).

Vincent, J. L., Snyder, A. Z., Fox, M. D., Shannon, B. J., Andrews, J. R., Raichle, M. E., \& Buckner, R. L. (2006). Coherent spontaneous activity identifies a hippocampal-parietal memory network. J Neurophysiol, 96(6), 3517-3531. doi:10.1152/jn.00048.2006

Vrba, J., \& Robinson, S. E. (2001). Signal processing in magnetoencephalography. Methods, 25(2), 249-271. doi:10.1006/meth.2001.1238

Wilson, M. A., \& McNaughton, B. L. (1994). Reactivation of hippocampal ensemble memories during sleep. Science, 265, 676-678.

Wolbers, T., \& Hegarty, M. (2010). What determines our navigational abilities? Trends Cogn Sci, 14(3), 138-146. doi:10.1016/j.tics.2010.01.001

Wu, X., \& Foster, D. J. (2014). Hippocampal replay captures the unique topological 
bioRxiv preprint doi: https://doi.org/10.1101/252288; this version posted January 23,2018 . The copyright holder for this preprint (which was not certified by peer review) is the author/funder, who has granted bioRxiv a license to display the preprint in perpetuity. It is made available under aCC-BY-NC-ND 4.0 International license.

structure of a novel environment. $J$ Neurosci, 34(19), 6459-6469.

2 doi:10.1523/JNEUROSCI.3414-13.2014

3

4 


\section{$1 \quad$ Figure legends}

2 Figure 1. Overview of the experiment. The upper panel shows environments of the two

3 conditions. The lower panel shows a flow chart of experimental procedure. In each training

4 set, there were 40 trials, including 20 hidden platform trials (the task was to find the hidden

5 platform in a pool with four cues) and 20 random swimming trials (the task was to aimless

6 swimming in a pool without visual cues and platform), which were alternatively presented (4

7 hidden platform trials, 4 random swimming trials, 4 hidden platform trials, 4 random

8 swimming trials...). The interval between each trial (ITI) was $4.5-5.5 \mathrm{~s}$, during which a grey

9 screen was presented and participants rested quietly without movement.

10 HP: hidden platform condition. RS: random swimming condition.

12 Figure 2. A. The mean of high-gamma power (pseudo- $Z$ values) during ITI ( $-4.5-0 \mathrm{~s})$ in the

13 right hippocampal ROI regions in the first and second training set for both post hidden

14 platform (Post HP) condition and post random swimming (Post RS) condition. B. The mean

15 of high-gamma power (pseudo- $Z$ values) during navigation period $(0-4 s)$ in the right

16 hippocampal ROI in the first and second training set for both hidden platform (HP) and

17 random swimming (RS) condition. C. Whole brain images of main effect of training set (peak

18 voxel in the right hippocampus: Talairach coordinates $\mathrm{x}=18 \mathrm{y}=-5 \mathrm{z}=-8$ ) and main effect of

19 condition (peak voxel in the parahippocampus: Talairach coordinates $x=27 y=-4 z=-24$ )

20 during ITI.

21 Error bar represents standard errors. * represents $\mathrm{p}<0.05$. 
1 Figure 3. The time frequency representations (TFRs) of the peak voxel in the right

2 hippocampus. The upper panel plots show the evolution of power change of right

3 hippocampal high-gamma during the ITI in first training set relative to the second one

4 collapsed across conditions (this shows the TFRs of the main effect of training set revealed by

5 ANOVA analyses) and the evolution of power change of right hippocampal high-gamma

6 during the ITI in post hidden platform condition relative to the random swimming condition

7 collapsed across training set (this shows the TFRs of the main effect of condition revealed by

8 ANOVA analyses). The lower panel shows right hippocampal high-gamma power change of

9 one individual participant. The black rectangular shows high-gamma $(80-140 \mathrm{~Hz})$ increase

10 during ITI as revealed by SAM beamformer analysis.

12 Figure 4. A. High-gamma power increase (pseudo-Z values differences) in the post hidden

13 platform condition during rest relative to that in the hidden platform condition during

14 navigation in the first training set (new environment) in the right hippocampal ROI (x-axis) of

15 each participant plotted against his learning rate during navigating in the second training set

16 (familiar environment) (y-axis). B. High-gamma power increase (pseudo- $Z$ values differences)

17 in the post hidden platform condition during rest relative to that in the hidden platform

18 condition during navigation in the second training set (familiar environment) in the right

19 hippocampal ROI (x-axis) of each participant plotted against his learning rate during

20 navigation in the second training set (familiar environment) (y-axis). The y-axis of Figure 4B

21 is the same as that of Figure 4A. 
1 Figure 5. A. The mean of theta power (pseudo- $Z$ values) in the right hippocampal ROI during

2 navigation in the hidden platform condition in the time window of $1.25-2.25 \mathrm{~s}$ when there

3 was an environmental encoding effect as shown in Pu et al. (2017) plotted against the mean of

4 high-gamma power (pseudo- $Z$ values) in the same region during ITI of $-2.5--0$ s (the time

5 window showed the strongest high-gamma increase in the group-averaged time frequency

6 representations in Figure 3) after navigation in the hidden platform condition. B. The mean

7 of theta power (pseudo- $Z$ values) in the right hippocampal ROI during navigation in the time

8 window of $1.25-2.25 \mathrm{~s}$ when there was an environmental encoding effect as shown in Pu et

9 al. (2017) plotted against the mean of high-gamma power (pseudo- $Z$ values) in the same

10 region during the ITI after navigation in the random swimming condition. C. Whole brain

11 images of the correlation between the mean of high-gamma power (pseudo- $Z$ values) in the

12 right hippocampal $\mathrm{ROI}$ in the time window of $-2.5-0 \mathrm{~s}$ and theta power (pseudo- $Z$ values) in

13 the time window of $1.25-2.25 \mathrm{~s}$ in each voxel across the whole brain. Threshold is set at

$14 \mathrm{p}<0.005$ (uncorrected). The local maximum is at right hippocampus (peak voxel: Talairach

15 coordinates $x=26, y=-17, z=-8$ ). 


\section{Experimental environments}

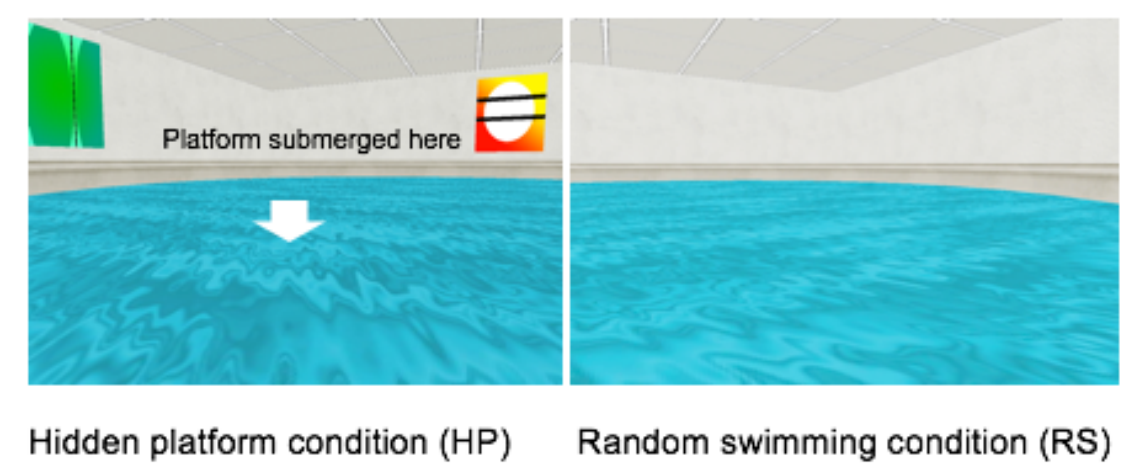

\section{Experimental procedures}

$1^{\text {st }}$ training set

Trial 1: HP

ITI: Post HP

Trial 2: HP

ITI: Post HP

$\because$

Trial 5:RS

ITI: Post RS

Trial 6:RS

ITI: Post RS

Trial 40:RS $2^{\text {nd }}$ training set

Trial 1: HP
ITI: Post HP
Trial 2: HP
ITI: Post HP
$\vdots$
Trial 5:RS
ITI: Post RS
Trial 6:RS
ITI: Post RS
$\vdots$
Trial 40:RS




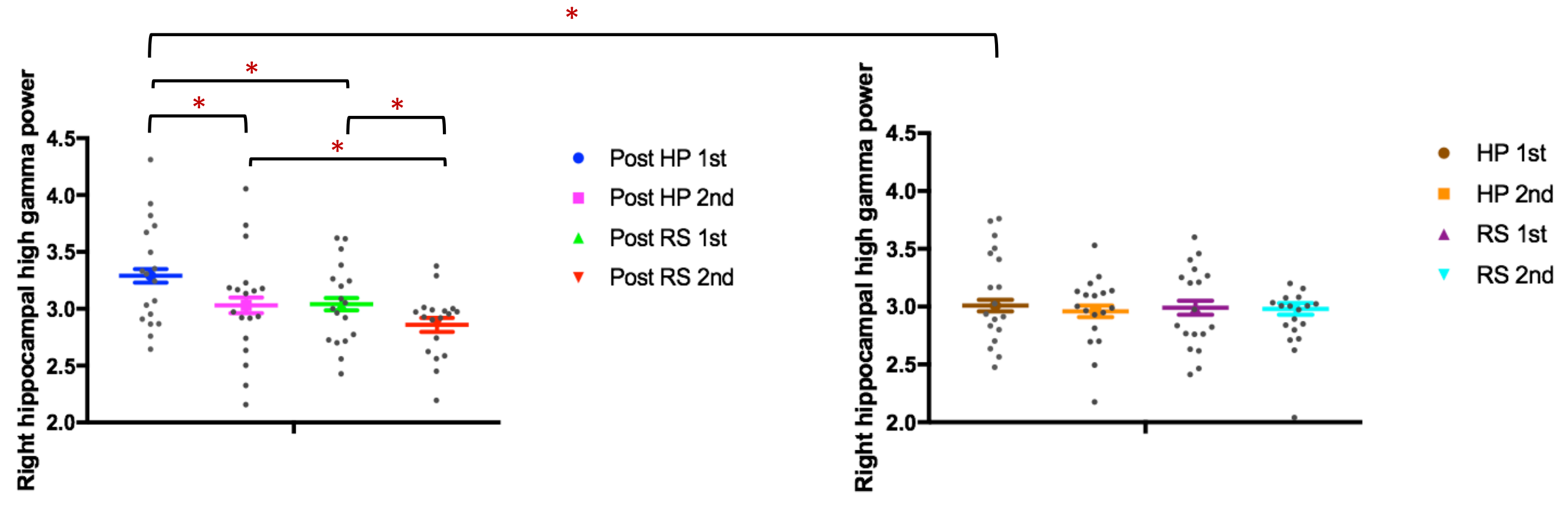

C. Whole brain images of significant effects of high-gamma power during ITI

Main effect of training set

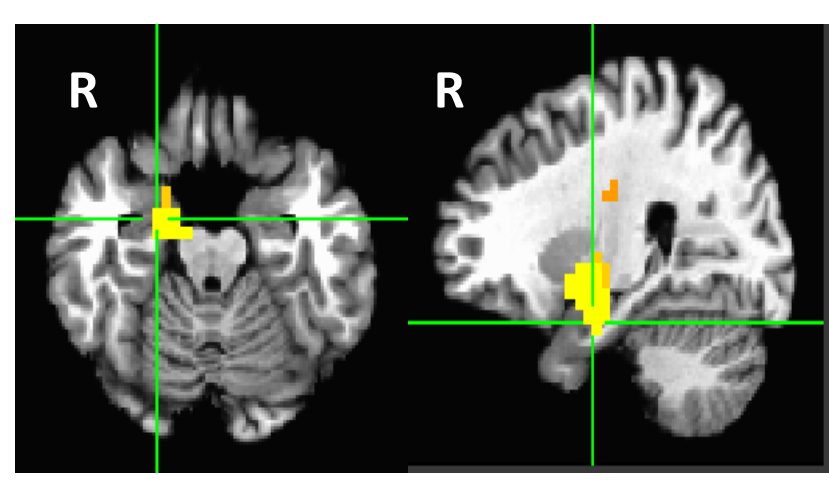

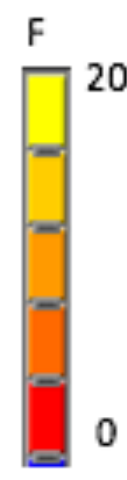

Main effect of condition

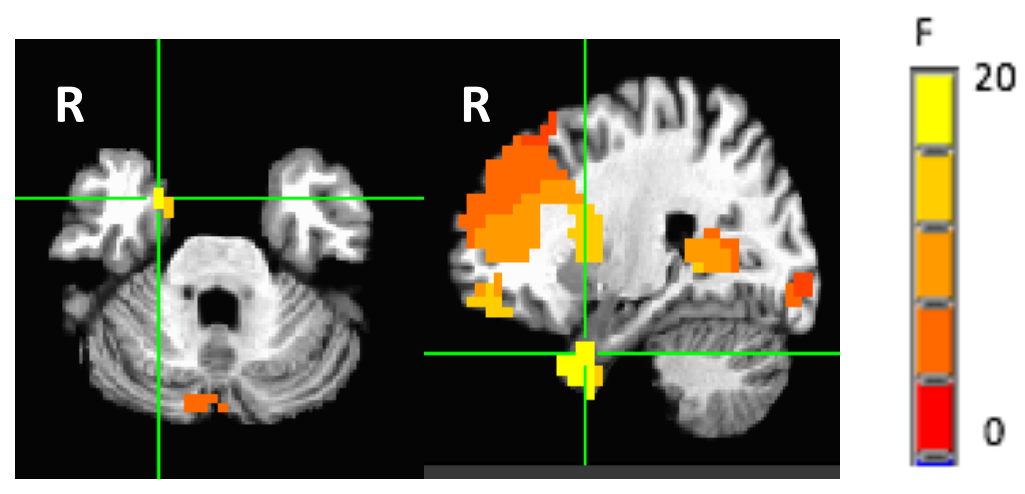


The first vs. second training set

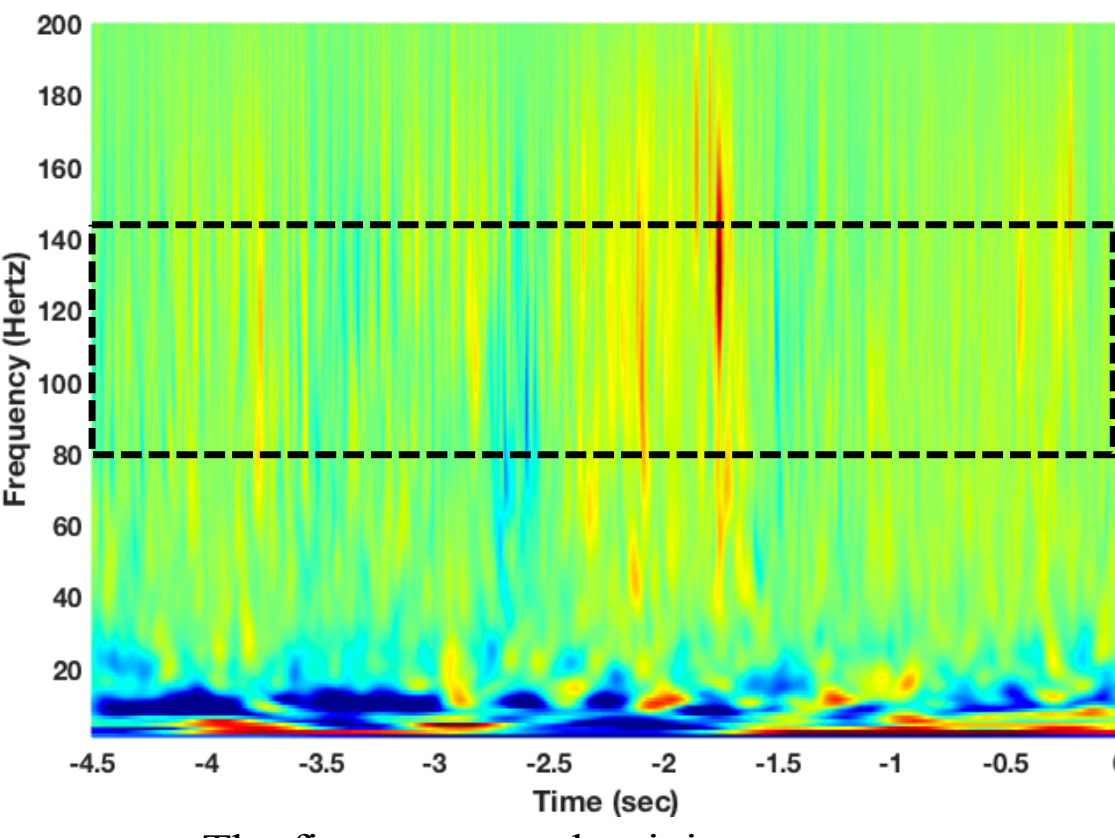

The first vs. second training set

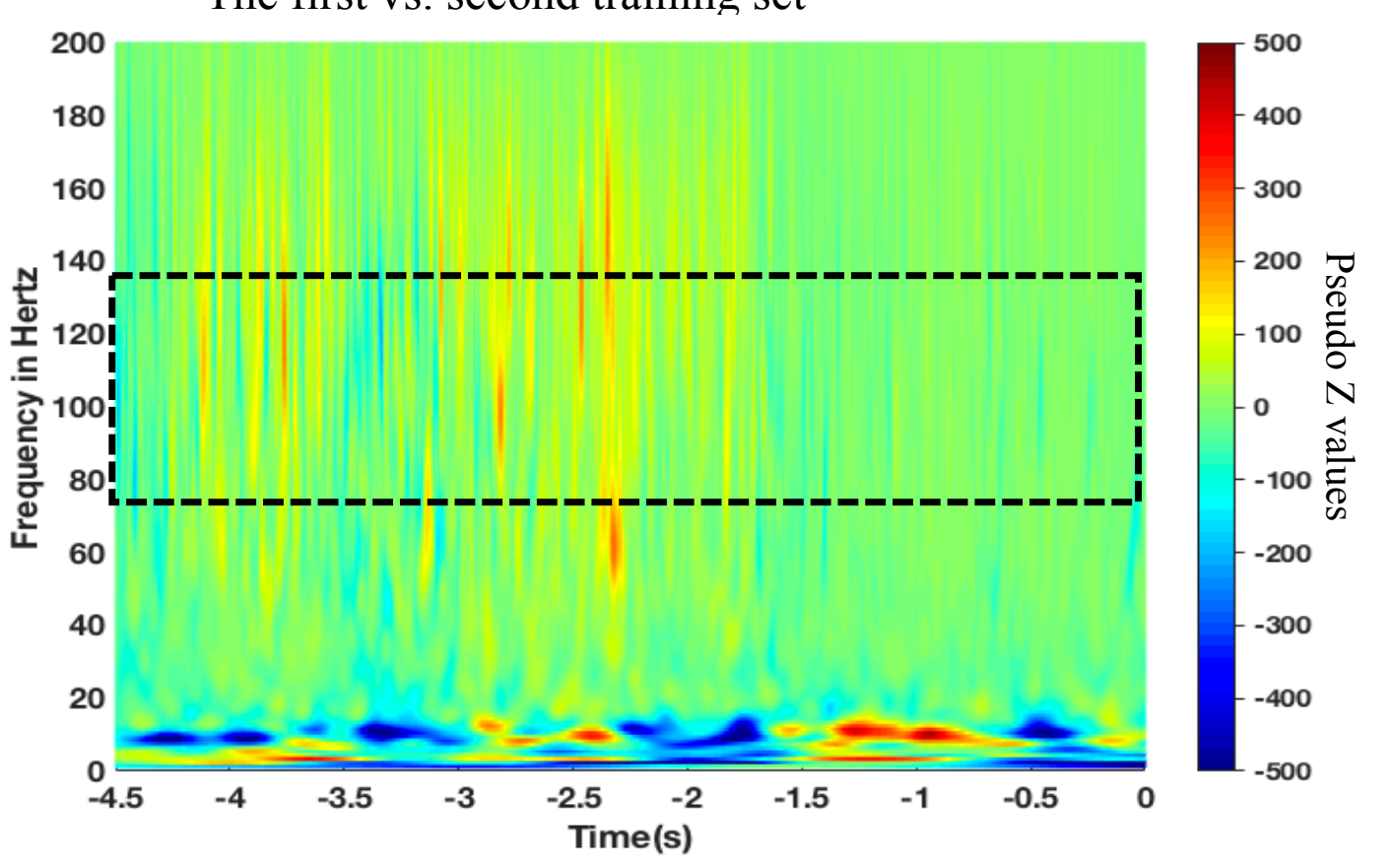

post hidden platform condition vs. post random swimming condition

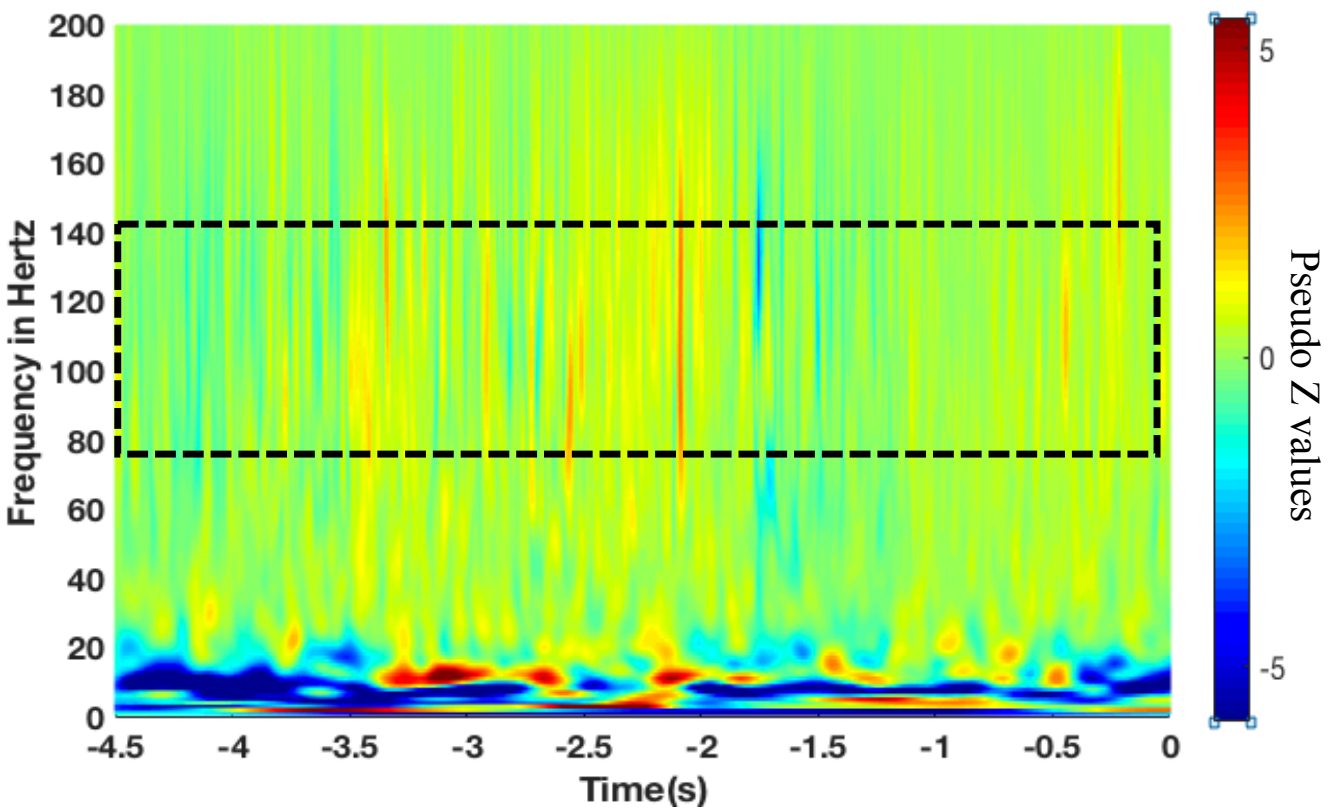

post hidden platform condition vs. post random swimming condition

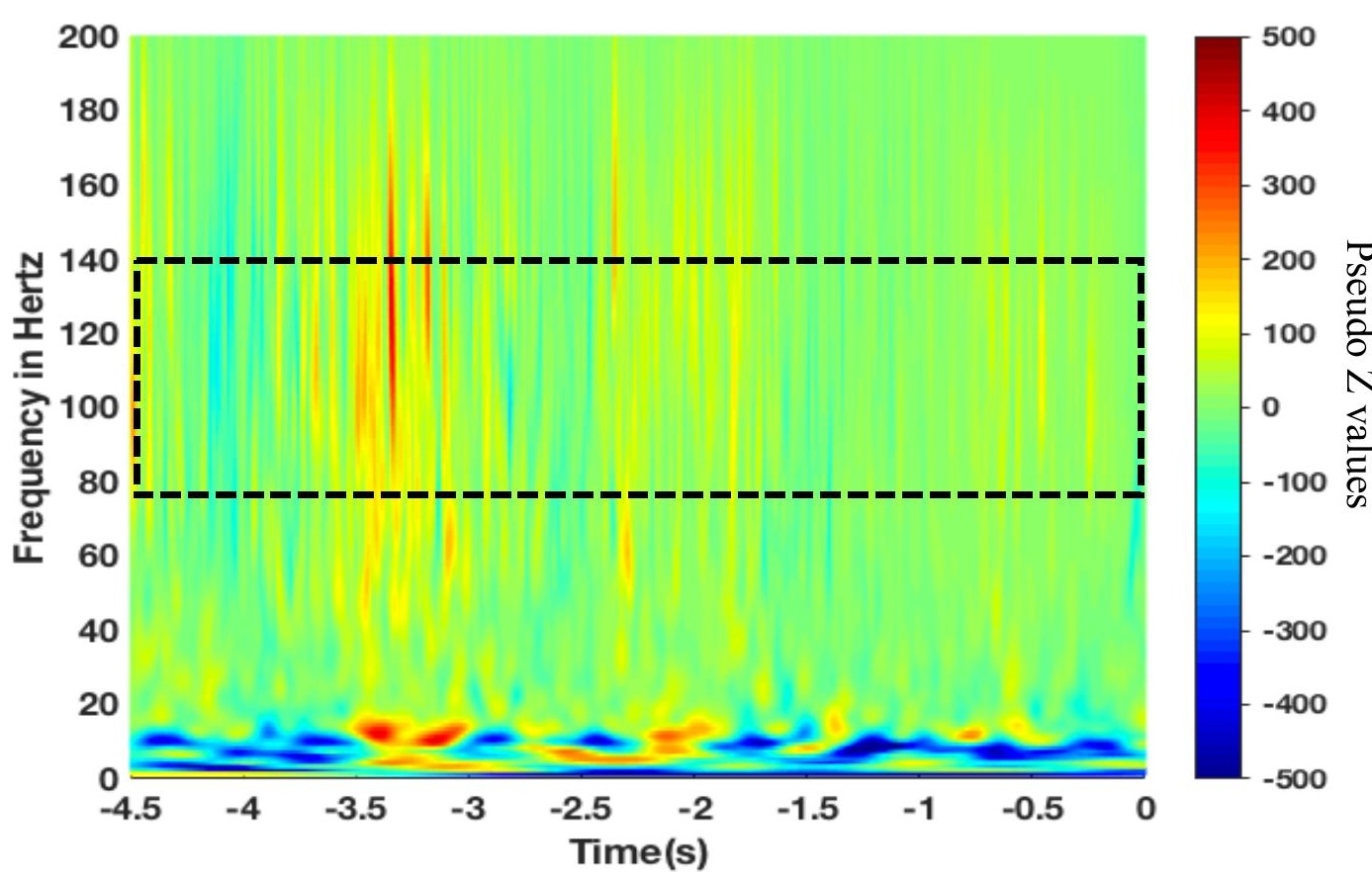


A.

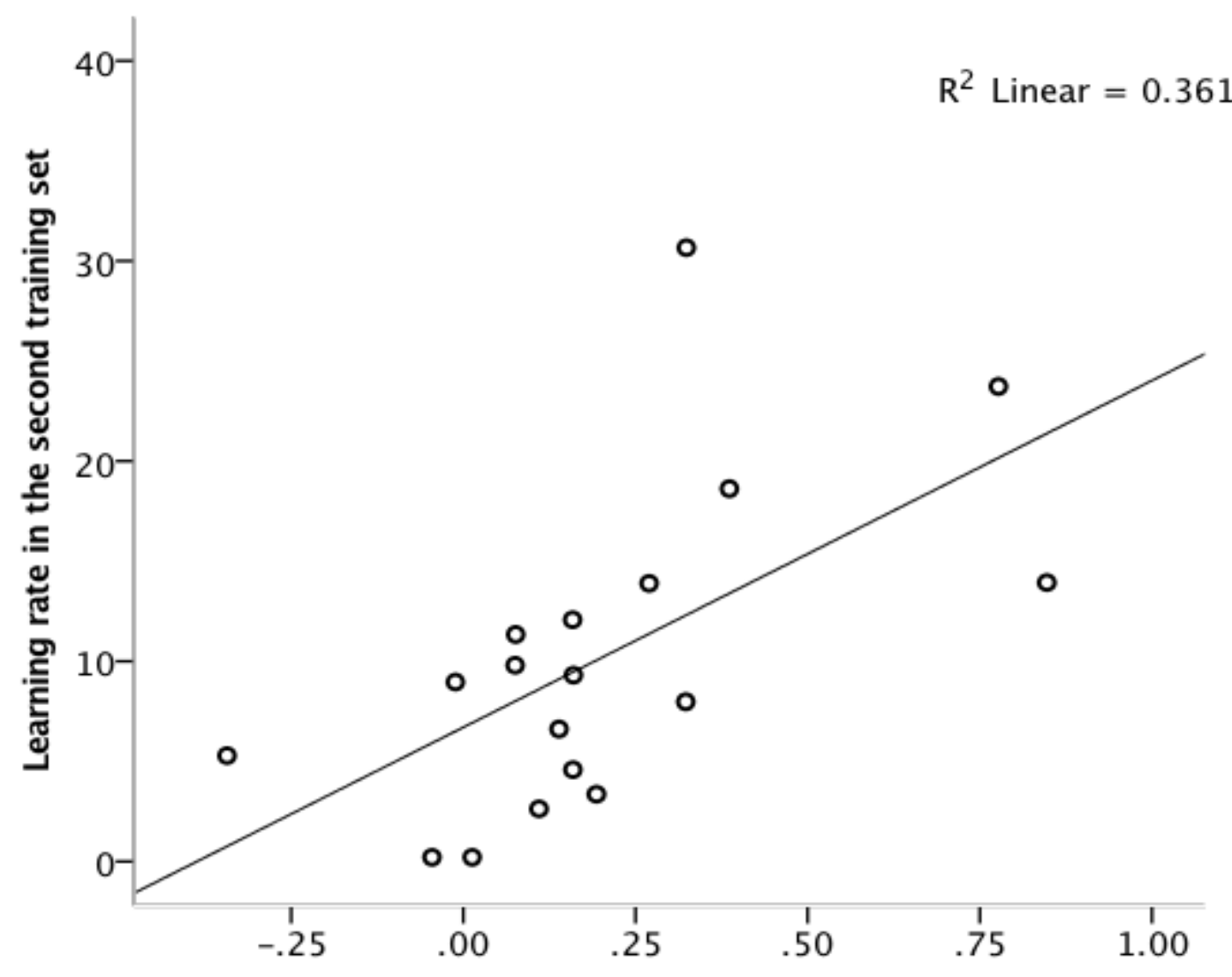

Right hippocampal high gamma power increase during ITI relative to navigation period in hidden platform condition in the first training set
B.

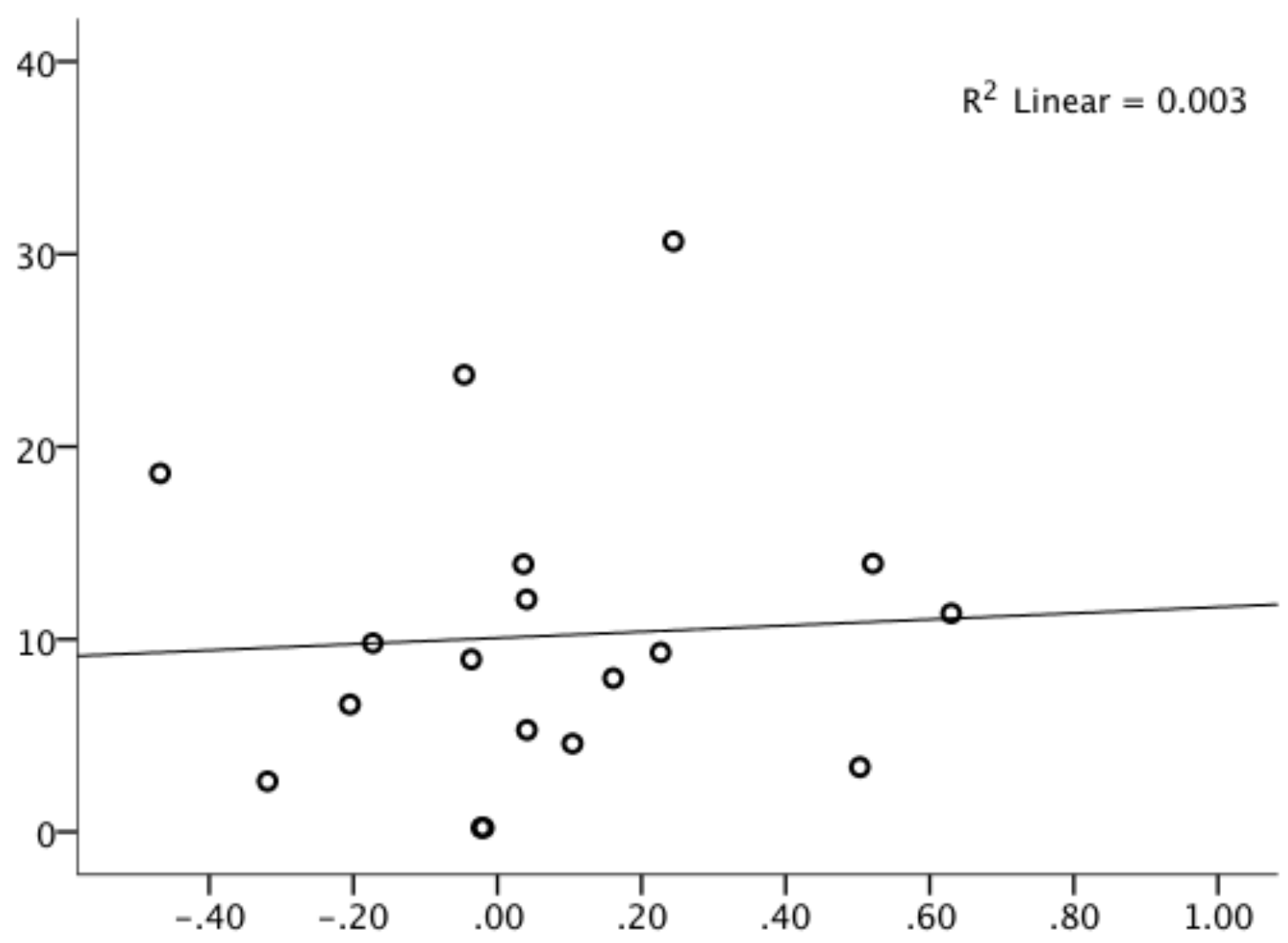

Right hippocampal high gamma power increase during $\mathrm{mI}$ relative navigation period in hidden platform condition in the second training set 
